\title{
Glutamate Uptake System in the Presynaptic Vesicle: Glutamic Acid Analogs as Inhibitors and Alternate Substrates*
}

\author{
Harry C. Winter ${ }^{1}$ and Tetsufumi Ueda ${ }^{2,3}$
}

(accepted March 23, 1992)

\begin{abstract}
A variety of naturally occurring amino acids, their isomers, and synthetic analogs were tested for their ability to inhibit uptake of $\left[{ }^{3} \mathrm{H}\right]$ glutamate into presynaptic vesicles from bovine cerebral cortex. Strongest inhibition $\left(\mathrm{K}_{\mathrm{i}}<1 \mathrm{mM}\right)$ was observed for trans-1-aminocyclopentane-1,3-dicarboxylic acid (t-ACPD) and erythro-4-methyl-L-glutamic acid (MGlu), while 4-methylene-L-glutamic acid (MeGlu) was only moderately inhibitory $(\mathrm{Ki}=\sim 3 \mathrm{mM})$, indicating that the synaptic vesicle glutamate translocator has higher affinity for trans-ACPD and MGlu than for glutamate. A few other amino acids, e.g., 4-hydroxyglutamic acid, S-carboxyethyl cysteine, and 5-fluorotryptophan, were slightly inhibitory; all L- and DL-isomers of protein amino acids and longer chain acidic amino acids were without measurable inhibition. Potassium tetrathionate and S-sulfocysteine exhibited strong to moderate noncompetitive or irreversible inhibition. Inhibition by t-ACPD, MGlu, or MeGlu was competitive with glutamic acid. Each of these competitive inhibitors was also taken up by the vesicle preparation in an ATP-dependent manner, as indicated by their being recovered unchanged from filtered vesicles. Similar results were obtained with reconstituted vesicles, while glutamate uptake by partially purified rat synaptosomes was inhibited only by MGlu. These results indicate that the glutamate translocator of presynaptic vesicles has stringent structural requirements distinct from those of the plasma membrane translocator and the metabotropic type of postsynaptic glutamate receptor. They further suggest possible structural requirements of pharmacologically significant compounds that can substitute for glutamic acid in the presynaptic side of glutamatergic synapses, thus serving to moderate or control glutamate excitation and associated excitotoxic effects in these neurons.
\end{abstract}

KEY WORDS: Synaptic vesicles; glutamate; ACPD; neurotransmitters.

\section{INTRODUCTION}

It is now widely accepted that glutamate functions as a major excitatory neurotransmitter in the vertebrate

${ }^{1}$ Department of Biological Chemistry, Medical School, and Mental Health Research Institute, The University of Michigan, Ann Arbor, MI 48109.

${ }^{2}$ Department of Pharmacology, Medical School, and Mental Health Research Institute, The University of Michigan, Ann Arbor, MI 48109.

${ }^{3}$ Address reprint requests to: Dr. Tetsufumi Ueda, Mental Health Research Institute, The University of Michigan, 205 Zina Pitcher Place, Ann Arbor, MI 48109-0720.

* Special issue dedicated to Dr. Paul Greengard.
CNS and that it is involved in memory and learning and in a variety of pathophysiologies in the brain (for reviews, ref. 1-2). There is increasing evidence that synaptic vesicles play a role in this process $(3-4) . \mathrm{Ca}^{2+}$. dependent release of glutamate from a non-cytosolic pool $(5,6)$ and ATP-dependent uptake of glutamate into highly purified, isolated synaptic vesicles (7-10) have been shown to occur. The vesicular glutamate uptake is driven by an electrochemical proton gradient that is generated by a proton-pump Mg-ATPase, and stimulated by physiologically relevant concentrations of chloride $(8-11,30)$. The key position of the vesicular uptake system in determin- 
ing the function of glutamate as a neurotransmitter suggests an interest in pharmacological means by which vesicular uptake can be modulated. Previous studies reported that a few close analogs of glutamate inhibited its uptake, apparently by acting as a competitive substrate (8); however, strongest competitive inhibition was observed by the peptide-containing ergot bromocriptine (12), although the competitive nature of this inhibition has recently been questioned (13). Recently, sulfur amino acids analogous to glutamate have also been reported to be inhibitors (14). In this communication, we further examine the specificity of the glutamate uptake system, and demonstrate for the first time that certain close analogs of glutamate are similarly transported, while other apparently inhibitory compounds probably act at other sites, possibly in the Mg-ATPase proton pump.

\section{EXPERIMENTAL PROCEDURE}

Purified bovine synaptic vesicles were prepared as previously described (15). The preparations typically had specific activities of glutamate uptake of $400-1000 \mathrm{pmol} / 10 \mathrm{~min} / \mathrm{mg}$ protein when assayed with $0.05 \mathrm{mM}$ glutamate. ATP-independent glutamate uptake was typically less than $10 \%$ of the ATP-dependent uptake in the absence of $\mathrm{Na}^{+}$ ion. Bovine synaptic vesicle preparations were solubilized and reconstituted according to the method of Carlson et al. (12); the reconstituted fraction had approximately twice the specific activity of the original vesicles. Synaptosomes were prepared from rat cerebral cortex by the method of Cotman (17).

Glutamate uptake was assayed in vesicle preparations essentially as described previously (8). Reaction tubes containing the test amino acid and vesicles were preincubated at $30^{\circ} \mathrm{C}$ for $5 \mathrm{~min}$ prior to addition of $\mathrm{L}-\left[\mathrm{G}-{ }^{3} \mathrm{H}\right]$ glutamate and ATP. Incubations were also carried out in the absence of ATP. After $1.5 \mathrm{~min}$ incubation, the reactions were stopped by addition of cold $0.15 \mathrm{M} \mathrm{KCl}$ and filtered as usual. Filters were then shaken with $7 \mathrm{ml}$ liquid scintillation cocktail for at least 1 hr and counted. Those values observed in the absence of ATP were subtracted from values observed for the complete system to obtain the ATP-dependent uptake activity. For measurement of uptake of unlabeled amino acids, the filters were shaken overnight with $80 \%$ ethanol in water, followed by centrifugation to remove the residue of the filter and washing of the residue. More than $95 \%$ of ${ }^{3} \mathrm{H}$-glutamate radioactivity taken up by vesicles was extracted from the filters by this procedure. The combined supernatant and wash was evaporated to dryness. For some experiments, this residue was redissolved in water and passed through a small $(0.6 \times 1.5 \mathrm{~cm})$ column of Dowex-1 acetate. The column was washed with $4 \mathrm{ml}$ water, eluted with $2 \mathrm{mI}$ of $2 \mathrm{~N}$ acetic acid, and the eluent evaporated to dryness. The Dowex-1treated samples were cleaner and easier to filter after derivatization, but there was no difference in the chromatograms of the acidic amino acids. Dried residues from either treatment were derivatized with PITC ${ }^{2}$,

\footnotetext{
2 Abbreviations used: PITC, phenylisothiocyanate; PTC, phenylthiocarbamyl; ACPD, 1-aminocyclopentane-1,3-dicarboxylic acid (cis or trans DL); MeGlu, 4-methyleneglutamic acid (racemate or unspecific optical isomers); MGlu, 4-methylglutamic acid (unspecified diastereo- or optical isomers).
}

and the PTC amino acid separated and quantitated by the Waters Picotag system. Substituted glutamic acids were resolved from the common protein amino acids by this technique (18). We observed, however, that PTC- $\alpha$-alkyl-substituted amino acids (i.e., $\alpha$-methyl glutamic acid and isomers of ACPD) initially gave peaks eluting between 2 and 3 minutes, in a position consistent with PTC derivatives of acidic amino acids. However, in the normal Pico-tag sample diluent, this derivative gradually converted to a product having characteristic elution times greater than 6 minutes; after 3-4 hours, only the later eluting peak was observed, whereas amino- and imino-acid PTC derivatives with an $\alpha$ hydrogen atom are completely stable under these conditions. Therefore, for analysis of ACPD, PITC-derivatized samples were dissolved in diluent and allowed to stand $4 \mathrm{hr}$ at room temperature before chromatographic analysis.

Amino acids, 4-Methylene-L-glutamic acid was isolated from germinating peanut plants as previously described (18); its DL racemate, as well as 4-methyl-DL-glutamic acid, was prepared synthetically (19). Erthyro-4-methyl-L-glutamic acid (2S, $4 \mathrm{R}$ configuration) was prepared enzymatically from racemic 4-methyl-2-oxoglutarate (prepared by $\mathrm{Cu}^{2+}$-catalyzed oxidative deamination of 4-methyl-DL-glutamic acid [20]) using asparate and pig heart glutamate:oxalacetate aminotransferase (EC 2.6.1.1), and terminating the reaction after the fast phase of transamination (21). Initially, cis- and trans-ACPD were purchased as racemic mixtures from Tocris Neuramin, UK. Larger quantities were subsequently synthesized via a Strecker synthesis (22) from 3carboxycyclopentanone synthesized by the method of Hope (23). The synthetic material was analyzed and resolved into cis and trans racemates by ion exchange chromatography on a modified Beckman $120 \mathrm{C}$ amino acid analyzer, using the purchased samples as standards. Structures of ACPD isomers and the enzymatically synthesized 4-methylL-glutamic acid were confirmed by NMR analysis.

Cysteine-S-sulfonic acid (S-sulfocysteine) was synthesized from cysteine and potassium tetrathionate (24), the latter being prepared by the method of Trudinger (25). To prepare S-sulfocysteine in the absence of tetrathionate, the method of Clark as described by Greenstein and Winitz (26) was used. The amino acid product from these two procedures was identical as judged by ion exchange and thin-layer chromatography, although the product of the former procedure likely contained small amounts of tetrathionate.

Other amino acids tested were commercially available preparations from various suppliers. Reagents for amino acid analysis were from Pierce Chem. Co.; L- [G- $\left.{ }^{3} \mathrm{H}\right]$ glutamate $(20-40 \mathrm{Ci} / \mathrm{mmol})$ was from Amersham; scintillation cocktail (Cytoscint) was from ICN; other reagents were highest quality grade from various suppliers.

\section{RESULTS}

Inhibition of $\left[{ }^{3} \mathrm{H}\right]$-glutamate uptake into synaptic vesicles by various naturally occurring and synthetic amino acids is shown in Table $\mathrm{I}$. At the lowest concentration used $(2.5 \mathrm{mM})$, only the three acidic amino acids having the same charged groups in the same relative position as glutamate showed inhibition of $50 \%$ or more. When a lower but definite level of inhibition was observed, additional concentrations of the compound were tested; 5-fluorotryptophan and S-sulfocysteine showed concentration-dependent inhibition, although inhibition comparable to the substituted glutamates was seen only 
Table I. Inhibition of Glutamate Uptake in Synaptic Vesicles by Substituted Glutamic Acids

\begin{tabular}{lcc}
\hline Amino acid added & $\begin{array}{c}\text { Conc. } \\
\mathrm{mM}\end{array}$ & $\begin{array}{c}\text { ATP-depd. uptake } \\
\text { \% of control }\end{array}$ \\
\hline None (control) & - & 100 \\
trans-ACPD & 2.5 & 19.0 \\
cis-ACPD & 2.5 & 86.8 \\
$e$-4-Methyl-DL-glutamate & 2.5 & 44.7 \\
e-4-Methyl-L-glutamate & 2.5 & 16.0 \\
4-Methylene-DL-glutamate & 2.5 & 69.9 \\
4-Methylene-L-glutamate & 2.5 & 52.0 \\
t-4-Mcthyl-DL-glutamate & 5.0 & 40.0 \\
e-4-Hydroxy-DL-glutamate & 5.0 & 62.0 \\
t-4-Hydroxy-DL-glutamate & 5.0 & 75.0 \\
S-Carboxyethyl-L-cysteine & 5.0 & 78.0 \\
S-Carboxymethyl-L-cysteine & 5.0 & 89.4 \\
4-Hydroxy-4-methyl-DL-glutamate & 5.0 & 90.0 \\
5-Fluorotryptophan & 2.5 & 78.5 \\
& 10.0 & 39.8 \\
5-Hydroxytryptophan & 2.5 & 97.6 \\
& 10.0 & 83.2 \\
\hline
\end{tabular}

Reaction mixtures $(0.1 \mathrm{ml})$ contained $10 \mathrm{mM}$ K-HEPES (pH 7.4), 6 $\mathrm{mM} \mathrm{Mg}{ }^{2+}, 2 \mathrm{mM}$ EGTA, $0.2 \mathrm{M}$ sucrose, $4 \mathrm{mM} \mathrm{KCl}$, vesicles ( 80 $\mu \mathrm{g}$ protein); ATP, $6 \mathrm{mM}$; L-[G- $\left.{ }^{3} \mathrm{H}\right\}$ glutamate, $2 \mu \mathrm{Ci}$ at $0.05 \mathrm{mM}$, and the unlabelled amino acid indicated. The mixtures were incubated for $1.5 \mathrm{~min}$ at $30^{\circ} \mathrm{C}$, filtered, and the washed filters counted.

The following amino acids showed $<7 \%$ inhibition ( $>93 \%$ of control) at $2.5 \mathrm{mM}$ : DL- $\alpha$-aminoadipate, iminodiacetic acid, S-ethyl-4-thiomethyl-L-glutamate, S-benzyl-4-thiomethyl-DL-glutamate, isoglutamine, L-pyroglutamate, trans-ACPD $\gamma$-methyl ester, DL- or L-histidine, Llysine, L-arginine, L-ornithine, L-canavanine, L-citrulline, L-methionine, L-methionine sulfoxide, DL-threonine, DL-homoserine, thioproline, albizziin ( $\beta$-ureidoalanine), L-phenylalanine, L-tyrosine $(1 \mathrm{mM})$, L-tryptophan, $m$-fluorophenylalanine, $p$-fluorophenylalanine, L-alanine, aminooxyacetic acid.

at concentrations of about $10 \mathrm{mM}$. The former compound has not yet been examined further, while the inhibition by S-sulfocysteine is discussed below. Other glutamic acid analogs, homologs, and derivatives had previously been found to have no significant effect at a concentration of $5 \mathrm{mM}(8)$.

Inhibition by the glutamate analogs MeGlu, MGlu, and ACPD were examined in greater detail. Inhibition of glutamate uptake by each of these amino acids was found to be competitive with glutamate, with $\mathrm{Ki}$ values shown in Table II. Comparison of the $\mathrm{Ki}$ values for 4-methylene-L-glutamate and erythro-4-methyl-Lglutamate with the apparent Ki values for the racemic mixtures indicates that only the $\mathrm{L}$-isomer is active in each case. Since optically pure isomers of trans-ACPD were not available for this study, we have not determined the chiral specificity for this compound. Assuming that a
Table II. Kinetics of Inhibition by Substituted Glutamic Acids of Glutamate Uptake by Synaptic Vesicles

\begin{tabular}{lcc}
\hline Amino acid added & Inhibition & $\mathrm{Ki}, \mathrm{mM}$ \\
\hline 4-Methylene-DL-glutamate & Competitive & $>5$ \\
4-Methylene-L-glutamate & Competitive & $2.95 \pm 0.74(3)$ \\
e-4-Methyl-DL-glutamate & Competitive & $1.63 \pm 0.24(4)$ \\
e-4-Methyl-L-glutamate & Competitive & $0.73 \pm 0.22(3)$ \\
trans-ACPD & Competitive & $0.44 \pm 0.18(6)$ \\
L-Glutamate & - & $1.59(2)$
\end{tabular}

Reaction mixtures were the same as those in Table $I_{3}$ except that total glutamate concentration was varied from 0.05 to $2.0 \mathrm{mM}$ at two or three concentrations of the inhibitor between $0.25 \mathrm{mM}$ and $5.0 \mathrm{mM}$. Double reciprocal plots were linear. Ki values for the competitive inhibitors were estimated from replots of the slopes vs. inhibitor concentration. Numbers in parentheses indicate the number of separate experiments from which the standard errors were calculated. The " $\mathrm{Ki}$ " value for glutamate was calculated as apparent inhibition of uptake of $\left[{ }^{3} \mathrm{H}\right]$ glutamate at $0.05 \mathrm{mM}$ by added unlabeled glutamate, and is virtually identical with the observed $\mathrm{Km}$ value.

single isomer (probably the L-isomer) is the active inhibitor, its $\mathrm{Ki}$ would be one-half that of the observed value, or about $0.2 \mathrm{mM}$. Even as the racemate, transACPD is the best competitive inhibitor of glutamate uptake thus far identified among the glutamate analogs.

Inhibition of glutamate uptake by trans-ACPD and erythro-4-methyl-L-glutamate was also tested in reconstituted vesicles. After solubilization with detergent and reconstitution of the vesicles, approximately 2 -fold greater specific activity of glutamate uptake was observed; this uptake was inhibited to the same or slighlty greater extent as observed with intact vesicles by each of the inhibitory glutamate analogs (data not shown). The effect of the vesicular uptake inhibitors on $\mathrm{Na}^{+}$-dependent uptake of glutamate into rat brain synaptosomes was also tested. Table III shows that erythro-4-methyl-L-glutamate and asparate substantially inhibited the plasma membrane transport; in contrast, trans-ACPD or 4-methylene-L-glutamate exhibited only slight inhibitory effect. This result provides further evidence for distinct transport mechanisms and transporters for these two membrane transport systems.

Uptake of Glutamate Analogs Into Synaptic Vesicles. The glutamate analogs that inhibited the initial rate of glutamate uptake also reduced the maximal level of glutamate incorporation to the same extent (data not shown), suggesting that the analog is taken up and accumulated in the vesicle competitively with glutamate. We tested directly the inhibitory glutamate analogs for their ability to be taken up by the vesicles. For these measurements, the unlabelled glutamate analogs were 
Table III. Inhibition by Substituted Glutamic Acids of Glutamate Uptake by Synaptosomal Plasma Membranes

\begin{tabular}{lcc}
\hline Amino acid added & \multicolumn{2}{c}{$\mathrm{Na}^{+}$-depd. } \\
$\mathrm{pmol}^{1}$ & $\%$ \\
\hline None & 48.2 & {$[100]$} \\
trans-ACPD & 44.5 & 91.5 \\
e-4-Methyl-L-glutamate & 18.5 & 39.4 \\
4-Methylene-L-glutamate & 44.6 & 91.5 \\
Asparate & 21.5 & 45.0 \\
\hline
\end{tabular}

Rat brain synaptosomes ( $70 \mu \mathrm{g}$ protein) were incubated at $37^{\circ} \mathrm{C}$ in 0.2 $\mathrm{ml} \mathrm{Krebs-Ringer} \mathrm{buffer} \mathrm{(20mM} \mathrm{Tris-HEPES} \mathrm{pH} \mathrm{7.4,} 150 \mathrm{mM} \mathrm{NaCl}$, $6.2 \mathrm{mM} \mathrm{KCl}, 1.2 \mathrm{mM} \mathrm{Na}_{2} \mathrm{HPO}_{4}, 1.2 \mathrm{mM} \mathrm{MgSO}_{4}, 10 \mathrm{mM}$ glucose). Uptake was initiated by addition of $\left[{ }^{3} \mathrm{H}\right]$ glutamate $(1 \mu \mathrm{Ci}, \mu \mathrm{M})$ plus the test compound at $25 \mu \mathrm{M}$. After 3 minutes incubation, solutions were diluted and filtered as for vesicles, except that glass-fiber filters were used. Blanks with low $\mathrm{Na}^{+}$contained choline chloride in place of $\mathrm{NaCl}$.

${ }^{1}$ Determined based upon the dpm value of ${ }^{3} \mathrm{H}$-glutamate retained on the filters and the specific activity of $\left[{ }^{3} \mathrm{H}\right]$ glutamate used, and corrected for $\mathrm{Na}^{+}$-independent uptake

determined in extracts of the filtered vesicles incubated with ATP and each of the analogs (Table IV). Although the amount of the added glutamate analog detected is small relative to other amino acids and peptides present in the filter extract, each of these amino acids gave a characteristic peak of its PTC derivative in the HPLC analysis, and the amount detected was at least 3-fold greater when vesicles were incubated with ATP. We therefore conclude that these compounds are transported by the glutamate transporter in the synaptic vesicle membranes in a manner similar to that of glutamate itself. More detailed kinetic analysis is best done using these compounds labeled with ${ }^{3} \mathrm{H}$ to high specific radioactivity, and will await their preparation.

Inhibition of Vesicular Glutamate Uptake by SulfurContaining Compounds. We considered the possibility that amino acids having a sulfonate, sulfinate, or thiosulfonate group in place of the 4-carboxylate group of glutamate might be active analogs of glutamic acid in the vesicular uptake process. Preliminary results indicated, however, that L-cysteate was virtually inactive, while L-cysteine sulfinate and L-homocysteine sulfinate at $5 \mathrm{mM}$ inhibited glutamate uptake $15 \%$ and $30 \%$, respectively. Further studies with L-cysteine sulfinate indicated that it inhibited glutamate uptake competitively, but less than $50 \%$ at a level of $10 \mathrm{mM}$. We also examined S-sulfocysteine, which we initially prepared by thiolysis of tetrathionate with cysteine (24). The product so obtained was strongly inhibitory $(40 \%$ at $0.5 \mathrm{mM})$. However, that preparation may not have been free of residual tetrathionate. We found that $0.2 \mathrm{mM} \mathrm{K}$ tetrathionate inhibited vesicular glutamate uptake greater than $50 \%$. The inhibition was non-competitive, suggesting that tetrathionate, and probably the S-sulfocy-

Table IV. Uptake of Substituted Glutamic Acids By Synaptic Vesicles

\begin{tabular}{lccc}
\hline Amino acid added & $\begin{array}{c}\text { ATP } \\
\mathrm{mM}\end{array}$ & $\begin{array}{c}\text { Glutamate } \\
\text { in extract, pmol }\end{array}$ & $\begin{array}{c}\text { Added amino acid } \\
\text { in extract, } \text { pmol }^{1}\end{array}$ \\
\hline None & 0 & 56 & - \\
Glutamate $(2.0 \mathrm{mM})$ & 6 & 104 & - \\
& 0 & 88 & - \\
4-Methylene-L-glutamate (10 mM) & 0 & 559 & - \\
& 6 & n.d. & 130 \\
e-4-Methyl-DL-glutamate $(1.0 \mathrm{mM})$ & 0 & n.d. & 470 \\
& 6 & 148 & 58 \\
e-4-Methyl-DL-glutamate $(10 \mathrm{mM})$ & 0 & 92 & 457 \\
& 2 & n.d. & 169 \\
+ Glutamate $(5.0 \mathrm{mM})$ & 2 & n.d. & 446 \\
trans-ACPD (10 mM) & 0 & n.d. & 321 \\
& 2 & n.d. & 476 \\
+ Glutamate $(5.0 \mathrm{mM})$ & 2 & n.d. & 1588 \\
\end{tabular}

Reaction mixtures were the same as those in Table $I$, except that no radioactive glutamate was added. After 10 min of incubation, reactions were stopped and filtered in the usual manner. Filters were extracted with $3 \mathrm{ml} 80 \%$ ethanol. Ethanol extracts were dried, derivatized with PITC, and analyzed for PTC amino acids by the pico-tag system.

No 4-methyl- or 4-methyleneglutamic acid was detected in samples incubated in the absence of these compounds.

${ }^{2}$ n.d., not determined 
steine preparation containing residual tetrathionate, inhibits glutamate uptake through S-sulfonation of one or more essential thiol residues in the Mg-ATPase or glutamate transporter protein. The Mg-ATPase proton pump and/or glutamate transport has been shown to inactivated by the thiol reagent $\mathrm{N}$-ethylmaleimide (8).

S-Sulfocysteine was subsequently prepared by sulfitolysis of cystine, the product of which is free of tetrathionate. S-Sulfo-L-cysteine prepared by this route also inhibited vesicular glutamate uptake, but $50 \%$ inhibition required approximately $5 \mathrm{mM}$ of the inhibitor. Furthermore, the inhibition was still non-competitive and was prevented by dithiothreitol. While this work was in progress, a report concerning inhibition of vesicular glutamate uptake by sulfur-containing amino acids appeared (14). That work reported both D- and L-isomers of the sulfonate and sulfinate of cysteine and homocysteine to be inhibitory at $10 \mathrm{mM}$, with the D-isomers being more effective. Furthermore, D- and L-S-sulfocysteine were equally inhibitory, although the nature of the inhibition was not examined. The lack of enantiomeric specificity of S-sulfocysteine is consistent with our observation of the non-competitive nature of the inhibition, and suggests that this compound, like tetrathionate, also causes inhibition by sulfonation of an essential thiol residue, although somewhat less effectively than does tetrathionate, rather than by acting as a substrate analog. Our data with the L-isomers of other acidic cysteine derivatives are qualitatively consistent with the results of Dunlop et al. (14).

\section{DISCUSSION}

The results we report here provide the first evidence that those glutamate analogs which are competitive inhibitors of vesicular glutamate uptake are themselves taken up by this transport system. The cyclic analog trans$\mathrm{ACPD}$ is the most effective glutamate analog found thus far which is an alternate substrate in the vesicular uptake system. Although bromocriptine is an effective inhibitor at a much lower concentration (12), there is no evidence that bromocriptine binds specifically at the glutamate binding site, or that it is transported into the vesicle. In fact, evidence has recently been advanced that bromocriptine acts by dissipating the electrochemical proton gradient that drives the uptake process (13).

The inhibitory effect of the glutamate analogs tested here provides further evidence for the stringent requirements of the glutamate transporter protein for a substrate having two negative charges (carboxylate anions) and one positive charge (amino group) in the proper orien- tation. The specificity for trans-over cis-ACPD further defines the geometry of its active site. When the 1-carboxylate and 1-amino groups of the 4 isomers of $\mathrm{ACPD}$ are superimposed (with the carboxylate above the plane of the ring), the 3-carboxylate anion lies in one of the 4 positions attached to the symmetrically-opposite ring carbon atoms at positions 3 and 4 . For the trans-isomers, the 3-carboxylate lies below the plane of the ring, either to the right or left of the vertical plane of symmetry. Thus, the putative positive charge of the transporter responsible for binding the 3-carboxylate group must also lie on that side of the substrate binding domain. More precise location of this group may be determined by testing the enantiomers of trans-ACPD; these studies are in progress.

Although the cyclopentane analogs of glutamic acid are more rigid structures than are the open-chain compounds, the ring can assume different comformations. One probable ring conformation of trans-ACPD places the 3-carboxylate and 1-amino groups in close axial proximity, leading to ionic interaction between them, which involves a hydrogen bond bridging the carboxylate and amino groups. A manifestation of this structure should be its ability to lactamize to the corresponding pyrollidone carboxylate more readily than does glutamic acid. In this respect, erythro-4-methylglutamic acid also forms its cyclic lactam at a faster rate than does the threo- isomer (21), while 4-methyleneglutamine undergoes cyclic elimination of the amide group more slowly that does glutamine (18). In each of these cases, the more readily cyclizing isomer or compound is the better substrate or competitive inhibitor of vesicular glutamate uptake. We suggest, therefore, that the binding of glutamate to its transporter requires a partially folded, rather than an extended conformation, with a corresponding ionic, hydrogen-bonded structure. This requirement could account for the observed inhibition of glutamate transport by small, strongly ionic structures as the chloride ion which could disrupt the critical ionic interaction (8). Such a notion is also consistent with the observed lack of interaction of the transporter with small polar or nonpolar amino acids (e.g., alanine, homoserine, threonine); these amino acids should fit into the binding site and interact with at least two of the three postulated charged groups located in the active center of the transporter, but do not involve the $\alpha$-amino groups in an intramolecular hydrogen-bonded structure. Aspartate likewise exhibits no interaction with the glutamate transporter (8); although hydrogen bonding between the $\alpha$ amino and $\beta$-carboxyl groups could occur, the structure is more constrained than that of glutamate or trans-ACPD, and, as such, lactamization generally does not occur. A 
key parameter for further investigation of this hypothesis would be the precise rate of lactamization of any given glutamate analog relative to glutamate, compared with its ability to compete with glutamate in the uptake process.

ACPD is also a cyclic analog of $\alpha$-aminoadipic acid, but of opposite enantiomeric configuration to glutamate. However, neither isomer of $\alpha$-aminoadipate exhibits significant inhibitory activity in this system (8). In order for $\alpha$-aminoadipate to assume the cyclic conformation, the $\alpha$ - and $\delta$-hydrogen atoms of $\alpha$-aminoadipic acid would be in close proximity as a result of the $\alpha$ - and $\delta$-carbon atoms having to assume an eclipsed conformation around the $\beta-\gamma$ bond; this conformation would, however, be energetically very unfavorable.

The metabotropic glutamate receptor coupled to inositol phosphate/ $\mathrm{Ca}^{2+}$ signal transduction has also been shown to respond to trans-ACPD (27), and it has been cloned and characterized (28). Unlike the vesicle transporter, however, the metabotropic receptor is more sensitive to L-glutamate than to trans-ACPD, and exhibits strongest response to the glutamate agonist quisqualate, which is without effect in the vesicle transport system (8). Although certian similarities may exist, such as preference for trans- over cis-ACPD (27), the glutamate binding sites of the metabotropic receptor and the vesicular transporter must be dissimilar.

The compounds reported here to be inhibitory to vesicular glutamate uptake are themselves unlikely candidates for pharmacologically relevant specific modulators of vesicular glutamate uptake, since a) they probably do not cross the blood-brain barrier, and b) in some cases they are known to interact with postsynaptic receptors. As mentioned above, trans-ACPD is an agonist of the metabotropic glutamate receptor, and $e$-4-methylglutamic acid, a natural product found in various plant species, has been shown to cause paralysis when injected into the somatic neuromuscular junction of an insect, in a manner similar to glutamate or quisqualate (29). However, they provide further detailed information about the geometry of the active site(s) of the glutamate transporter in presynaptic vesicles. This information would be of use in designing more specific and potent inhibitors, and could aid in efforts to purify and further characterize the vesicular glutamate transport protein.

\section{ACKNOWLEDGMENTS}

Supported by U.S. Public Health Service, National Institutes of Health Grant NS 026107.

The authors express appreciation to Professor E.E. Dekker of the Department of Biological Chemistry for providing materials and space to carry out the chemical syntheses used in this work, to Drs. Joel Tabb and Phillip Kish for their help in the preparation of purified synaptic vesicles and helpful discussions, and to Mary Roth for assistance in preparing the manuscript.

\section{REFERENCES}

1. Monaghan, D. T., Bridges, R. J., and Cotman, C. W. 1989. The excitatory amino acid receptors: their classes, pharmacology, and distinct properties in the function of the central nervous system. Ann. Rev. Pharmacol. Toxicol. 29:365-402.

2. Watkins, J. C., Krogsgaard-Larsen, P., and Honore, T. 1990. Structure activity relationships in the development of excitatory amino acid receptor agonists and competitive antagonists. Trends Pharmacol. Sci. 11:25-33.

3. Ueda, T. 1986. Glutamate transport in the synaptic vesicle. Pages 173-195, in Roberts, P., Storm-Mathisen, J. $r$ and Bradford, H. F. (eds.), Excitatory Amino Acids, Macmillan, London.

4. McMahon, H. T., and Nicholls, D. G. 1991. The bioenergetics of neurotransmitter release. Biochim. Biophys. Acta 1059:243264.

5. Nicholls, D. G., and Sihra, T. S. 1986. Synaptosomes possess an exocytotic pool of glutamate. Nature 321:772-773.

6. Kish, P. E., and Ueda, T. 1991. Calcium-dependent release of accumulated glutamate from synaptic vesicles within permeabilized nerve terminals. Neurosci. Lett. 122:179-182.

7. Naito, S., and Ueda, T. 1983. Adenosine triphosphate-dependent uptake of glutamate into Protein I-associated synaptic vesicles. J. Biol. Chem. 258:696-699.

8. Naito, S., and Ueda, T. 1985. Characterization of glutamate uptake into synaptic vesicles. J. Neurochem. 44:99-109.

9. Maycox, P. R., Deckwerth, T., Hell, J. W., and Jahn, R. 1988. Glutamate uptake by brain synaptic vesicles. J. Biol. Chem. 263:15423-15428.

10. Cidon, S., and Sihra, T. S. 1989. Characterization of a $\mathrm{H}^{+}$-ATPase in rat brain synaptic vesicles: coupling to L-glutamate transport. J. Biol. Chem. 264:8281-8288.

11. Shioi, J., Naito, S., and Ueda, T. 1988. Glutamate uptake into synaptic vesicles of bovine cerebral cortex and electrochemical potential difference of proton across the membrane. Biochem. J. 258:499-504.

12. Carlson, M. A., Kish, P. E., and Ueda, T. 1989. Glutamate uptake into synaptic vesicles: competitive inhibition by bromo. criptine. J. Neurochem. 53:1889-1894.

13. Moriyama, Y., Amakatsu, K., Yamada, H., Park, M.-Y., and Futai, M. 1991. Inhibition of neurotransmitter and hormone transport into secretory vesicles by 2-(4-Phenylpiperdino)cyclohexanol and 2-Bromo- $\alpha$-ergocryptine: both compounds act as uncouplers and dissipate the electrochemical gradient of protons. Arch. Biochem. Biophys. 290:233-238.

14. Dunlop, J., Fear, A, and Griffiths, R. 1991. Glutamate uptake into synaptic vesicles - inhibition by sulphur amino acids. Neuroreport 2:377-379.

15. Kish, P. E., and Ueda, T. 1989. Glutamate accumulation into synaptic vesicles. Pages 9-25, in Fleischer, S., and Fleischer, B., (eds.), Methods in Enzymology, Vol. 174, Academic Press, New York.

16. Carlson, M. D., Kish, P. E., and Ueda, T. 1989. Characterization of the solubilized and reconstituted ATP-dependent vesicular glutamate uptake system. J. Biol. Chem. 264:7369-7376.

17. Cotman, C. 1974. Isolation of synaptosomal and synaptic plasma membrane fractions. Pages 445-452, in Fleischer, S., and Packer, L. (eds.), Methods in Enzymology, Vol. 31, Academic Press, New York.

18. Winter, H. C., Powell, G. K., and Dekker, E. E. 1988. 4-Methyleneglutamic acid and 4-methyleneglutamine: isolation from 
extracts of peanut seedlings and determination by high-performance liquid chromatography. Prep. Biochemistry 18:121-136.

19. Powell, G. K., and Dekker, E. E. 1981. A modified, high-yield procedure for the synthesis of unlabeled and 14C-labeled 4methylene-DL-glutamic acid. Prep. Biochemistry 11:339-350.

20. Dekker, E. E., and Maitra, U. 1962. Conversion of $\gamma$-hydroxyglutamate to glyoxylate and alanine: purification and properties of the enzyme system. J. Biol. Chem. 237:2218-2227.

21. Winter, H. C., and Dekker, E. E. 1987. An unusual distribution of 4-substituted glutamic acids in Sophora japonica. Phytochemistry $26: 2477-2480$.

22. Gass, J. D., and Meister, A. 1970. 1-Amino-1,3-dicarboxycyclohexane (cycloglutamic acid), a new glutamic acid analog and a substrate of glutamine synthetase. Biochemistry 9:842-846.

23. Hope, E. 1912. The condensation of ethyl sodiomalonate with ethyl citraconate and the synthesis of $\beta$-methyltricarballylic acid. J. Chem. Soc. Trans. 101:892-912.

24. Inglis, A. S., and Liu, T. Y. 1970. The stability of cysteine and cystine during acid hydrolysis of proteins and peptides. J. Biol. Chem. 245:112-116.
25. Trudinger, P. A. 1961. Thiosulfate oxidation and cytochromes in Thiobacillus $X$. Biochem. J. 78:680-686.

26. Greenstein, J. B., and Winitz, M. 1961. Pages 1913-1914, in Chemistry of the Amino Acids, John Wiley \& Sons, New York.

27. Schoepp, D. D., Johnson, B. G., Salhoff, C. R., McDonald, J. W., and Johnston, M. V. 1991. In vitro and in vivo pharmacology of trans- and cis-(+)-1-amino-1,3-cyclopentanedicarboxylic acid: dissociation of metabotropic and ionotropic excitatory amino acid receptor effects. J. Neurochem. 56:1789-1796.

28. Masu, M., Tanabe, Y., Tsuchida, K., Shigemoto, R., and Nakanishi, S. 1991. Sequence and expression of a metabotropic glutamate receptor. Nature 349:760-765.

29. Hart, R. J., Potter, C., Wright, R. A., and Lea, P. J. 1978. Relationship between the in vivo and in vitro activity of some naturally occurring glutamate analogues on the somatic neuromuscular junction of Lucilia sericata. Physiol. Entomology 3:289295.

30. Tabb, J. S., Kish, P. E., Van Dyke, R., and Veda, T. 1992. Glutamate transport into synaptic vesicles. J. Biol. Chem. 267 (in press). 